\title{
L-band search for substellar companions in the Tucana and $\beta$ Pictoris moving groups
}

\author{
M. Kasper ${ }^{1}$, D. Apai ${ }^{2}$, W. Brandner ${ }^{3}$, L.M. Close ${ }^{4}$ and K. Geißler ${ }^{3}$ \\ ${ }^{1}$ European Southern Observatory, Karl-Schwarzschild-Str. 2, 85748 Garching, Germany \\ email: mkasper@eso.org \\ ${ }^{2}$ NASA Astrobiology Institute \\ ${ }^{3}$ Max-Planck-Institut für Astronomie, Königstuhl 17, 69117 Heidelberg, Germany \\ ${ }^{4}$ Steward Observatory, University of Arizona, 933 N Cherry Ave., Tucson AZ 85721, USA
}

\begin{abstract}
Radial velocity surveys provide evidence that giant extrasolar planets are common, but their detection space is limited to only a few astronomical units from the stars. In order to close this gap, the adaptive optics assisted NIR imager NAOS-CONICA (NACO) at the VLT was used for a deep (15-20 minutes exposure time per target) L-band survey of a sample of closeby young stars. All stars are members of the Tucana and $\beta$ Pictoris moving groups apart from the the somewhat older star HIP 71395 that has a radial velocity trend suggesting a massive planet in large orbit. The chosen observation wavelength is very well suited for very high contrast imaging of close companions at this age and makes this survey unique. The goal was to detect substellar companions to these stars at distances as close as 5-20 AU and ultimately to detect giant extrasolar planets down to a few Jupiter masses, to measure their frequency, and - by comparison with models - determine their physical properties. This paper presents the results obtained on a subsample of 12 stars that have been observed during ESO P73.
\end{abstract}

Keywords. extrasolar planets, adaptive optics, L band, NACO, high contrast imaging.

\section{Introduction}

A major scientific breakthrough of the last years was the discovery of extra-solar planets by radial velocity searches (Mayor \& Queloz 1995) which add up to more than 100 such systems known to date (exoplanets.org). Although the sample is still statistically small, it appears that a significant fraction of stars (about 6\%) have planetary companions of relatively high mass at close-in orbit. The discovery space of radial velocity searches does not cover planets at a distance larger than a few AU, so few observational results on these objects exist to date. The recent detections of substellar (Liu 2002, McCaughrean et al. 2004) or even planetary mass (Chauvin et al. 2005, Neuhäuser et al. 2005) companions around young stars by direct imaging with AO let this technique appear most promising to close this gap.

Current programs searching for substellar companions are mostly carried out in Kband by broadband imaging (Masciadri et al. 2005) or in H-band using simultaneous observations in narrow $\mathrm{H}$-band filters inside and outside the methane absorption feature to efficiently subtract speckle noise (Close et al. 2005). However, the models of Baraffe et al. (2003) predict colors of $\mathrm{H}-\mathrm{L} \approx 3$ mag and $\mathrm{K}-\mathrm{L} \approx 4$ mag for a 5 Jupiter mass planets at the age of 100 Myr making the L-band an interesting observing window to search for substellar companions. For example, the 5 Jupiter mass companion to the 8 Myr old brown dwarf 2M1207 detected by Chauvin et al. (2005) is as red as K-L $\approx 1.75$ mag and $\mathrm{H}-\mathrm{L} \approx 3.81 \mathrm{mag}$. As additional advantages compared to shorter wavelengths, the L-band image quality delivered by $\mathrm{AO}$ is better and temporally more stable reducing speckle 


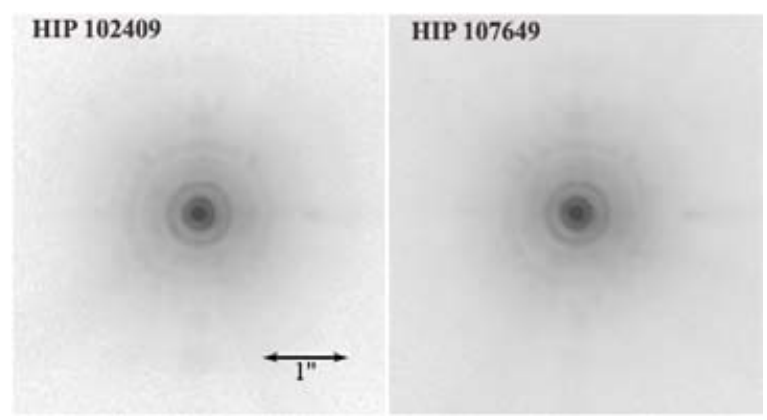

Figure 1. NACO L-band images of HIP 102409 and HIP 107649.

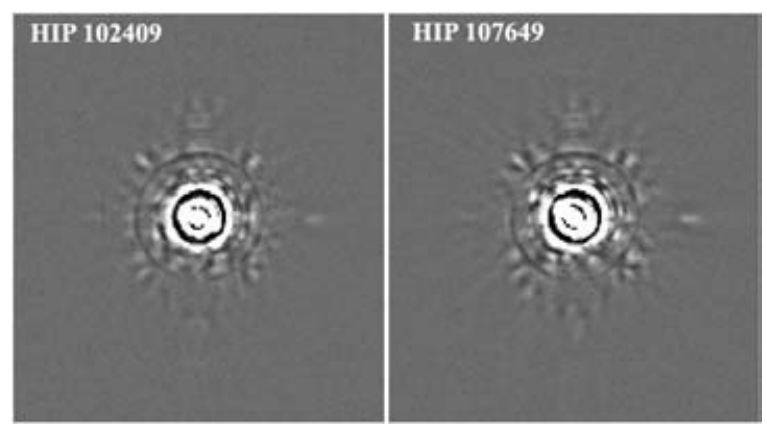

Figure 2. High-pass filtered images of HIP 102409 and HIP 107649.

Table 1. Target stars

\begin{tabular}{|c|c|c|c|c|c|c|}
\hline Target & $\begin{array}{l}\text { Spectral } \\
\text { type }\end{array}$ & $\begin{array}{l}\text { Distance } \\
\quad(\mathrm{pc})\end{array}$ & $\begin{array}{c}\mathrm{V} \\
(\mathrm{mag})\end{array}$ & $\begin{array}{c}\mathrm{L} \\
(\mathrm{mag})\end{array}$ & $\begin{array}{l}\text { Age } \\
(\mathrm{Myr})\end{array}$ & Remarks \\
\hline HIP 71395 & K0 & 17 & 7.6 & 5.6 & $500-1000$ & $\begin{array}{l}\text { lin. radial velocity trend } \\
\text { (Butler et al. } 2003 \text { ) }\end{array}$ \\
\hline HIP 116748 & G6IV & 46 & 8.5 & 6.9 & 30 & Tucana, $5.3^{\prime \prime}$ binary \\
\hline HIP 102141 & M4.5 & 10 & 11.3 & 5.8 & 12 & $\beta$ Pic, GJ799, 2.8" binary \\
\hline HIP 102409 & M0 & 10 & 8.9 & 5.2 & 12 & $\begin{array}{l}\beta \text { Pic, GJ803, debris disk } \\
\text { (Kalas et al. 2004) }\end{array}$ \\
\hline HIP 107649 & G0V & 16 & 5.6 & 4.2 & 30 & Potential Tucana member \\
\hline HIP 105441 & $\mathrm{~K} 2 \mathrm{~V}$ & 31 & 9 & 6.8 & 30 & Potential Tucana member \\
\hline HIP 105441B & $?$ & 31 & 10.5 & 8 & 30 & Potential Tucana member \\
\hline HIP 1993 & $\mathrm{~K} 7 \mathrm{~V}$ & 37 & 11.5 & 8.2 & 30 & Confirmed Tucana member \\
\hline HIP 3556 & M1.5 & 39 & 11.9 & 7.9 & 30 & Potential Tucana member \\
\hline HIP 2729 & $\mathrm{~K} 5 \mathrm{~V}$ & 46 & 9.6 & 6.7 & 30 & Confirmed Tucana member \\
\hline HIP 4448 & $\mathrm{~K} 4 \mathrm{~V}$ & 44 & 9 & 6.3 & 30 & Potential Tucana member \\
\hline HIP 1113 & G6V & 44 & 8.8 & 7.2 & 30 & Potential Tucana member \\
\hline HIP 1481 & G0V & 41 & 7.5 & 6.1 & 30 & Confirmed Tucana member \\
\hline
\end{tabular}

noise and facilitating PSF subtraction. All these advantages more than compensate for the increased sky background.

\section{Targets and observations}

The target sample has been compiled of 18 nearby (10-46 pc) young stars which are members of the Tucana association (Zuckerman et al. 2001) and the $\beta$ Pic moving group (Zuckerman et al. 2001) of which 12 were observed in ESO observing period P73 (2004) 


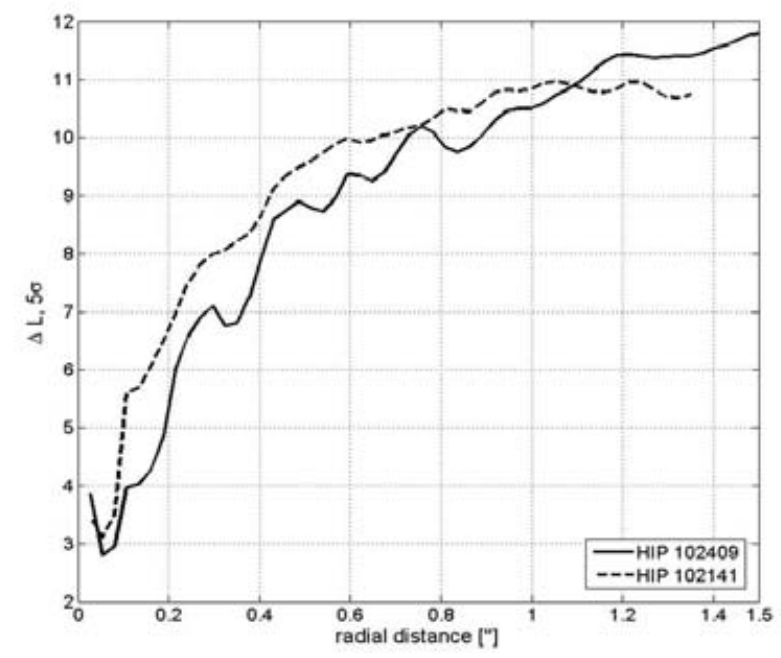

Figure 3. Contrast achieved on HIP 102409 and HIP 102141.

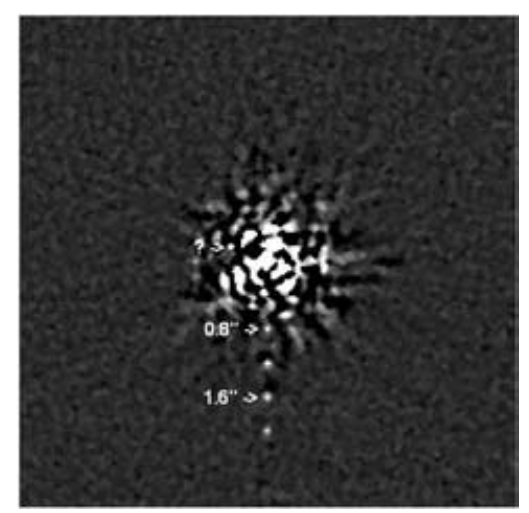

Figure 4. Difference of HIP 102409 and HIP 102141 with artificial test planets (10 mag fainter).

and are listed in table 1 . The ages of the member stars were estimated to be between 10 and 30 Myr (e.g., Kastner et al. 2003, Stelzer \& Neuhäuser 2001) providing an ideal test bed for detecting extra-solar planets and study their formation. The sample was restricted to the later spectral types $\mathrm{G}, \mathrm{K}$, and $\mathrm{M}$. Considering the reported V-band luminosities and the colors according to the spectral types, the targets in our sample have estimated L-band luminosities between $\mathrm{L}=4.2 \mathrm{mag}$ and $\mathrm{L}=8.2 \mathrm{mag}$ which are reported in table 1 .

A 100 Myr old, 5 Jupiter mass planet would have luminosities between L = 14.06. mag at $10 \mathrm{pc}$ and $\mathrm{L}=17.07 \mathrm{mag}$ at $40 \mathrm{pc}$ (Baraffe et al. 2003). The $5 \sigma$ L-band point source detection limit of NACO in 15-20 minutes exposures is permitting the detection of planetary mass companions for all observed stars.

All targets have been observed in the $\mathrm{L}^{\prime}$ filter centered at $3.8 \mu \mathrm{m}$ with the $\mathrm{L} 27$ camera of CONICA providing a pixel scale of 27 mas. NAOS was counter-chopping, i.e. the field selector of the wavefront sensor followed the motion of the telescope's M2 providing AO 

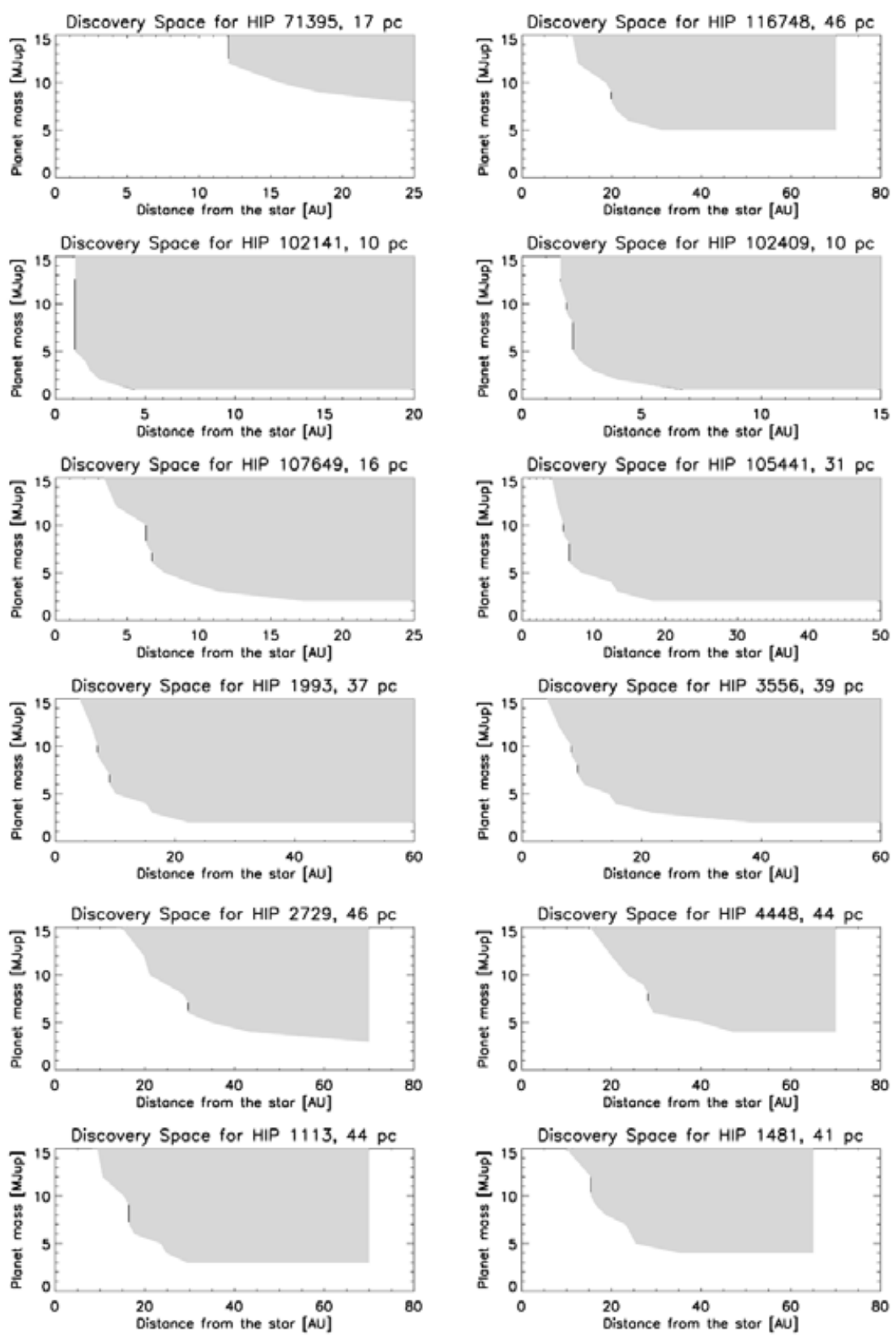

Figure 5. Discovery space for the observed targets.

corrected images at both chopping positions effectively doubling the observing efficiency. Total on-source exposure times for all shown data sets are between 15 and 20 minutes. The targets were observed in pairs that were chosen such that position on sky and brightness of the partners were as close as possible. In this way, the objects could be used to calibrate or subtract each others PSF and no additional observing of calibrator stars was necessary. 
Figure 1 shows HIP 102409 and HIP 107649 observed for 15 minutes on source each and separated by about 30 minutes from each other. The image quality is remarkably good (Strehl ratio of about $70 \%$. The PSF core was saturated so the Strehl ratio estimate is based on the intensity in the Airy rings and is pretty coarse) and stable permitting efficient PSF calibration and subtraction.

\section{Data analysis}

After standard image processing (flat fielding, bad pixel cleaning, sky subtraction), the image pairs were superimposed with subpixel accuracy, normalized and high-pass filtered. The high-pass filtering was done by subtracting from the image the median filtered $(\approx 3$ FWHM box-width) image. This procedure efficiently eliminates large scale structure while leaving point sources (such as substellar companions) relatively unaffected. Figure 2 shows a high-pass filtered version of figure 1. Finally the images have been median filtered with a small box width to reduce pixel-to-pixel variations.

Contrast curves were calculated from the difference of a pair of final images that had been observed together by calculating the standard deviation of the pixel intensity in 2 pixel wide annuli of variable radii centered on the stars. Figure 3 plots the contrast at five standard deviations as a function of the angular distance from the stars for two of the observed pairs, i.e., 4 objects. The solid line represents the pair HIP 102409 and HIP 107649 shown in figures 1 and 2. The dashed line shows the contrast of the $2.8^{\prime \prime}$ binary HIP 102141. The latter is an interesting case, because the close binary permits very efficient speckle subtraction due to the simultaneous observation. Hereby, angular anisoplanatism is negligible due to the small separation of the stars which is at least 10 times smaller than the anisoplanatic angle of atmospheric turbulence in L-band. The better contrast of about one magnitude at small angular separations indicates the gain achievable by simultaneous observation. The flattening of the curves at larger angular distance is due to the sensitivity hitting the background limit.

A mandatory verification of predicted contrast levels is the insertion of fake planets into the images before performing the data analysis. Figure 4 shows the difference of the two images shown in figure 2 with added fake planets at distances of $0.8^{\prime \prime}, 1.2^{\prime \prime}, 1.6^{\prime \prime}$ and $2^{\prime \prime}$. Comparing this image with the predicted contrast shown in figure 3 , the $5 \sigma$ criterion is obviously just enough to suspect a detection. Another interesting result is that the added planets are point-like while most speckles show a different mostly elongated morphology. Masciadri \& Raga (2004) have developed a method to use this result in order to better discriminate the planets from the speckles. The question mark denotes a non-elongated feature in the image that would be worth further investigations.

\section{Results}

Knowing the stars age, brightness and distance, the calculated radial contrast for the individual objects can be converted into a discovery space using using planetary evolutionary models by Baraffe et al. (2003). The discovery space describes the detectable planet mass as a function of separation from the star in AU and is shown for all target stars in figure 5. Since no clear companion candidates were found, the discovery space provides upper limits on planet masses for a given separation from the host stars. Given the contrast curves are comparable to the ones achieved using other methods such as Kband imaging or H-band differential imaging and the contrast gain of one magnitude and more in L-band due to the smaller brightness difference of potential substellar companions to their host stars, the discussed observations are among the most sensitive observations to date. 


\section{Acknowledgements}

We thank the ESO VLT astronomers for carrying out our observations in service mode. This material is partly based upon work supported by the National Aeronautics and Space Administration through the NASA Astrobiology Institute under Cooperative Agreement No. CAN-02-OSS-02 issued through the Office of Space Science.

\section{References}

Baraffe, I., Chabrier, G., Barman, T.S., Allard, F. \& Hauschildt, P.H. 2003, A\& A 402, 701

Chauvin, G., Lagrange, A.-M., Dumas, C., Zuckermann, B., Mouillet, D., Song, I., Beuzit, J.-L. \& Lowrance, P. 2005, A\&BA 438L, 25

Close, L.M., Lenzen, R., Guirado, J.C., Nielsen, E.L., Mamajek, E.E., Brandner, W., Hartung, M., Lidman, C. \& Biller, B. 2005, Nature 433, 286

Kalas, P., Liu, M.C. \& Matthews, B.C. 2004, Science 303, 1990

Kastner, J.H., Crigger, L., Rich, M. \& Weintraub, D.A. 2003, ApJ 585, 878

Liu, M.C., Fischer, D.A., Graham, J.R., Lloyd, J.P., Marcy, G.W., Butler \& R.P. 2002, ApJ 571,519

Masciadri, E. \& Raga, A. 2004, ApJ 611, 137

Masciadri, E., Mundt, R., Henning, Th. \& Alvarez, C. 2004, ApJ 611, 137

Mayor, M. \& Queloz, D. 1995, Nature 378, 355

McCaughrean, M.J., Close, L.M., Scholz, R.-D., Lenzen, R., Biller, B., Brandner, W., Hartung, M. \& Lodieu, N. 2004, A\&A 413, 1029

Neuhäuser, R., Guenther, E.W., Wuchterl, G., Mugrauer, M., Bedalov, A., Hauschildt, P.H. 2005, A\& A 435, 13

Stelzer, B. \& Neuhäuser, R. 2001, A\&A 538, 377

Zuckerman, B., Song, I., Bessell, M.S. \& Webb, R.A. 2001, ApJ 562, 87

Zuckerman, B., Song, I. \& Webb, R.A. 2001, ApJ 559, 388 